\title{
BINARY RECYCLED PULSARS AS THE MOST PRECISE PHYSICAL LABORATORY
}

\author{
G. S. Bisnovatyi-Kogan \\ Space Research Institute RAN, 84/32, Profsoyuznaya St., Moscow, RU-117997, Russia, \\ Joint Institute for Nuclear Research, Bogoliubov Laboratory of Theoretical Physics, Dubna, Russia
}

(Received December 25, 2006)

The following problems are discussed. 1. Pulsars and close binaries. 2. Hulse-Taylor pulsar. 3. Disrupted pulsar pairs. 4. Recycled pulsar statistics. 5. Enhanced evaporation: formation of a single recycled pulsar. 6. General relativity effects: neutron star + neutron star. 7. A Double pulsar system. 8. Checking general relativity. 9. Variability of the gravitational constant. 10. Space Watch.

Key words: pulsars, gravitation, precise time.

PACS number(s): 97.60.Gb, 95.30.Sf

\section{INTRODUCTION}

The discovery of pulsars was announced by Hewish, Bell et al. [1]. Until 1973 all the known pulsars (more than 100) had been single, while more than a half of massive stars (predecessors of pulsars) are in binaries. Possible explanations of this fact had been suggested: all pairs are disrupted during the explosion, or there is no possibility to form a radiopulsar in pairs [2].

\section{PULSARS AND CLOSE BINARIES}

X-ray satellite UHURU was launched in 1970, and soon after that X-ray pulsars in binaries had been discovered. One of the best studied is X-ray pulsar Her X-1. It has the pulsation period of $P_{p} \approx 1.24 \mathrm{~s}$, orbital period $P_{\text {orb }} \approx 1.7$ days, neutron star mass about $1.4 M_{\odot}$, and optical star with a mass about $2 M_{\odot}$, see e.g. [3].

It was shown in [4] that this system should give birth to the binary radiopulsar by the following reasons. 1 . After 100 million years the optical star will become a white dwarf, mass transfer will be finished, and the system will be transparent to radio emission. 2. X-ray pulsar is accelerating its rotation due to accretion, so after the birth of the white dwarf (WD), the neurton star (NS) will rotate rapidly, with $P_{p}$ about $100 \mathrm{~ms}$. It was suggested in [4] that binary radiopulsars had not been found until 1973 , because the magnetic field of the neutron star is decreasing about 100 times during the accretion, so binary radiopulsars are very faint objects. Pulsar luminosity $L \sim B^{2} / P^{4}$, so at small $B$, luminosity $L$ is low even at the rapid rotation. Magnetic field is decreasing due to screening by the infalling plasma.

\section{HULSE-TAYLOR PULSAR}

Hulse and Taylor discovered [5] the first binary radiopulsar with a period $P_{p}=0.05903 \mathrm{~s}$, orbital period $P_{\text {orb }} \approx 7.75 \mathrm{~h}$, orbit eccentricity $e=0.615$ (see Fig. 1 ). The properties of the first binary pulsar coincide with our predictions: rapid rotation together with a small magnetic field $[6,7]$

$$
\begin{gathered}
\dot{E}=-I \Omega \dot{\Omega}=I\left(\frac{2 \pi}{P_{p}}\right)^{2} \frac{\dot{P}_{p}}{P_{p}}=2 \cdot 10^{33} \mathrm{erg} / \mathrm{s} \\
B_{p}^{2}=\left(\frac{3 I c^{3} P_{p} \dot{P}_{p}}{8 \pi^{2} R_{\mathrm{NS}}^{6}}\right)^{1 / 2} \approx 2.3 \cdot 10^{10} \mathrm{Gs} .
\end{gathered}
$$

The average magnetic fields of single radiopulsars is about $10^{12}$ Gauss.

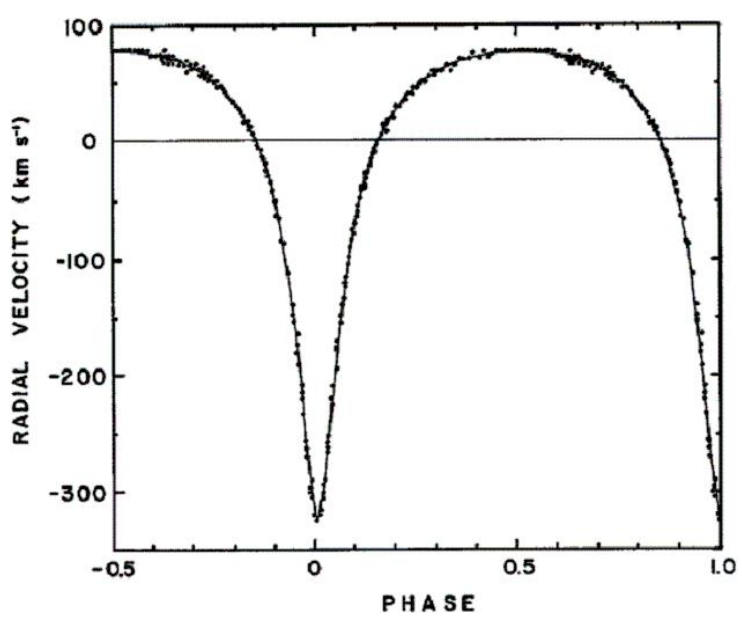

Fig. 1. Velocity curve for the first binary pulsar. Points represent measurements of the pulsar period distributed over parts of 10 different orbital periods. The curve corresponds to the pulsar parameters from the text.

\section{DISRUPTED PULSAR PAIRS}

Suggestions for separation of pulsars at birth were made in [4] where 10 pairs of single pulsars with a possible origin from one pair had been listed (see Table. 1). The same idea was recently considered by Vlemmings etal. [8], who measured pulsar proper motions. They suggested a common origin of the pair B2020+28 and 
B2021+51 (see Fig. 2). It is interesting that one of these pulsars $(2020+28)$ had been suggested in [4] for the common origin with another pulsar $(2016+28$, line 8 in Table. 1). The last pulsar is much closer to B2020+28 on the sky, and both are situated at the same distance [9]. The pulsar B2021+51 is much farther on the plane, and almost two times by the distance, but velocities intersect in Cygnus OB association.

\begin{tabular}{|c|c|c|c|c|c|c|c|c|c|}
\hline No & PSR & R. A. & Decl & $l^{\mathrm{II}}$ & $b^{\mathrm{II}}$ & $P_{s}$ & $\mathrm{DM} \frac{\mathrm{pc}}{\mathrm{cm}^{3}}$ & $\dot{P} \frac{\mathrm{s}}{\mathrm{day}}$ & $\tau=\frac{P}{\dot{P}} \mathrm{yr}$ \\
\hline 1 & P0943+10 & $9^{\mathrm{h}} 43^{\mathrm{m}} 20^{\mathrm{s}}$ & $10^{\circ} 05^{\prime} 33^{\prime \prime}$ & 225.4 & 43.2 & 1.098 & 15 & - & - \\
\hline & P0950+08 & 95031 & 080943 & 228.9 & 43.7 & 0.253 & 3 & 0.0198 & $3.5 \cdot 10^{7}$ \\
\hline 2 & $\mathrm{P} 0809+74$ & $\begin{array}{llll}8 & 09 & 03\end{array}$ & 743810 & 140 & 31.6 & 1.30 & 6 & 0.014 & $2.5 \cdot 10^{8}$ \\
\hline & $\mathrm{P} 0904+77$ & 904 & 7740 & 135.3 & 33.7 & 1.58 & - & - & - \\
\hline 3 & P1700-18 & 170056 & -18 & 4.0 & 14.0 & 0.802 & $\leq 40$ & 0.154 & $6.9 \cdot 10^{6}$ \\
\hline & P1706-16 & 170633 & -163721 & 5.8 & 13.7 & 0.653 & 25 & 0.55 & $3.3 \cdot 10^{6}$ \\
\hline 4 & $\mathrm{P} 0329+54$ & 32911 & 542438 & 145.0 & -1.2 & 0.714 & 27 & 0.177 & $1.1 \cdot 10^{7}$ \\
\hline & $\mathrm{P} 0355+54$ & 35500 & 5413 & 148.1 & 0.9 & 0156 & 55 & - & - \\
\hline 5 & $\mathrm{P} 0525+21$ & 52545 & 215532 & 183.8 & -6.9 & 3.7455 & 51 & 3.452 & $3 \cdot 10^{6}$ \\
\hline & $\mathrm{P} 0531+21$ & 53131 & 215655 & 184.6 & -5.8 & 0.03313 & 57 & 36.526 & $2.5 \cdot 10^{3}$ \\
\hline 6 & P1426-66 & 142634 & -660994 & 312.3 & -6.3 & 0.787 & 60 & - & - \\
\hline & P1449-65 & 144922 & -65 & 315.3 & -5.3 & 0.180 & 90 & - & - \\
\hline 7 & P1845-01 & 1845 & -0127 & 31.3 & 0.2 & 0.660 & 90 & - & - \\
\hline & P1845-04 & 184510 & -040532 & 28.9 & -1.0 & 0.598 & 142 & - & - \\
\hline 8 & P2016+28 & 201600 & 283031 & 68.1 & -4.0 & 0.558 & 14.16 & 0.01 & $1.2 \cdot 10^{8}$ \\
\hline & $\mathrm{P} 2020+28$ & 202033 & 284430 & 68.9 & -4.7 & 0.343 & - & - & - \\
\hline 9 & $\mathrm{P} 2111+46$ & 211141 & 4636 & 89.1 & -1.2 & 1.015 & 141.4 & - & - \\
\hline & $\mathrm{P} 2154+40$ & 215456 & 4000 & 90.5 & -11.5 & 1.525 & 110 & - & - \\
\hline 10 & $\mathrm{P} 0611+22$ & 061110 & 2235 & 188.7 & 2.4 & 0.335 & 99 & - & - \\
\hline & $\mathrm{P} 0540+23$ & 054010 & 2330 & 184.4 & -3.3 & 0.246 & 72 & - & - \\
\hline
\end{tabular}

Table 1. Single pulsars of possible common origin, from [4].

\section{RECYCLED PULSAR (RP) STATISTICS}

Recycled pulsars is a separate class of neutron stars containing more than 180 objects. All passed the stage of accreting pulsars accelerating the rotation and decreasing the magnetic field. So we have ordinary pulsars with $P_{p}=0.033 \div 8 \mathrm{~s}, B=10^{11} \div 10^{13} \mathrm{Gs}$; and recycled pulsars with $P_{p}=1.5 \div 50 \mathrm{~ms}, B=10^{8} \div 10^{10}$ Gs. These two groups are distinctly visible on the $P-\dot{P}$ diagram (Fig. 3).

\section{ENHANCED EVAPORATION: FORMATION OF SINGLE RP}

Most recycled pulsars consist of pairs NS + WD, and single NS, about 180 objects in total. Only $6 \mathrm{NS}+\mathrm{NS}$ pairs exist, like Hulse--Taylor pulsar. Single recycled pulsars (small $P_{p}$ and $B$ ) have passed the stage of X-ray pulsar in binary, and later had lost the WD companion. Besides, NS + NS are in the Galaxy disk, and NS $+\mathrm{WD}$, and single are in the galactic bulge, and in the globular clusters. Globular clusters (GC) contain 0.001 of the mass of the Galaxy, and about $1 / 2$ of the recycled pulsars. Formation of the binary in GC may happen by tidal capture or triple collision. Disruption of the binary RP also happens by collisions with GC stars: most single RP are situated in GC. In the most dense GC we have [10]: $31 \mathrm{RP}$ in Terzian $5(11-\mathrm{BP}, 10+10$ ? - single); $22 \mathrm{RP}$ in 47 Tuc $(14-\mathrm{BP}, 7+1$ ? - single). In total: $80 \mathrm{RP}$ in Gal (15 - single), $108 \mathrm{RP}$ in GC $(40+15$ ? - single).

Simple disruption of the pair by collisions with field stars does not work, because hard pairs become even harder by collisions. When a pair is in the state of a disk accretion, with WD filling its Roche lobe (Fig. 4) situation is opposite, and also hard pairs become softer during collisions due to enhanced mass transfer, and finally the pair is disrupted. This process named "enhanced evaporation", first considered in [11], could explain the formation of a single RP in GC, and their appearance in bulge may be connected with a full evaporation of GC (see also [12]).

\section{GENERAL RELATIVITY EFFECTS: NS+NS}

In the NS+NS binary tidal effects are negligibly small, so several post-Keplerian (PK) general relativity (GR) effects had been measured from pulsar timing. They include [13] parameter $\dot{\omega}$, determining the rate of relativistic apse line motion, parameter $\gamma$, representing the amplitude of the signal time delay due to variable gravitational redshift and time dilation (quadratic Doppler effect), when the pulsar moves in an elliptical orbit. The emission of gravitational waves results in the loss of the orbital angular momentum and decreases the orbital period $\dot{P}_{\text {orb }}$. The parameters $r$ and $s$ determine the time delay due to the Shapiro effect and are related to the companion's gravitational field. 


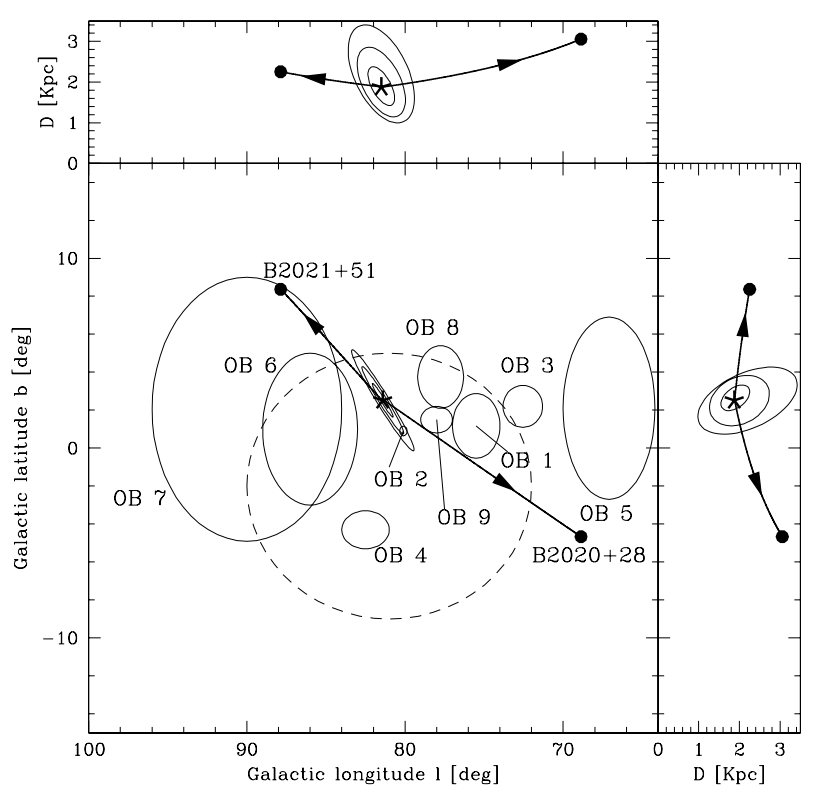

Fig. 2. The 3-dimensional pulsar motion through the Galactic potential for B2020+28 and B2021+51. The dashed circle represents the Cygnus superbubble, while the labelled solid ellipses are the Cygnus OB associations with positions and extents. The extent of OB 2 is unknown and only the centre of the association is indicated. The thick solid lines indicate the pulsar paths, with the origin denoted by the starred symbol and the arrows pointing in the direction of motion. The current positions are indicated by the solid dots. The elliptical contours around the pulsars' origin in these panels indicate the 1, 2 and $3 \sigma$ levels of the likelihood solution for the birth location, from [8].

$$
\begin{gathered}
\dot{\omega}=3 T_{\odot}^{2 / 3}\left(\frac{P_{\text {orb }}}{2 \pi}\right)^{-5 / 3} \frac{1}{1-e^{2}}\left(M_{A}+M_{B}\right)^{2 / 3}, \\
\gamma=T_{\odot}^{2 / 3}\left(\frac{P_{\text {orb }}}{2 \pi}\right)^{1 / 3} e \frac{M_{B}\left(M_{A}+2 M_{B}\right)}{\left(M_{A}+M_{B}\right)^{4 / 3}}, \\
\dot{P}_{\text {orb }}=-\frac{192 \pi}{5} T_{\odot}^{5 / 3}\left(\frac{P_{\text {orb }}}{2 \pi}\right)^{-5 / 3} \frac{\left(1+\frac{73}{24} e^{2}+\frac{37}{96} e^{4}\right)}{\left(1-e^{2}\right)^{7 / 2}} \\
\quad \times \frac{M_{A} M_{B}}{\left(M_{A}+M_{B}\right)^{1 / 3},} \\
r=T_{\odot} M_{B}, T_{\odot}=G M_{\odot} / c^{3}=4.925490947 \mu \mathrm{s}, \\
s=x T_{\odot}^{-1 / 3}\left(\frac{P_{\text {orb }}}{2 \pi}\right)^{-2 / 3} \frac{\left(M_{A}+M_{B}\right)^{2 / 3}}{M_{B}}
\end{gathered}
$$

NS + NS RPs are the best laboratories for checking of General Relativity. $1913+16$ timing had shown (indirectly) the existence of gravitational waves, Nobel Prize of Hulse and Taylor (1993).

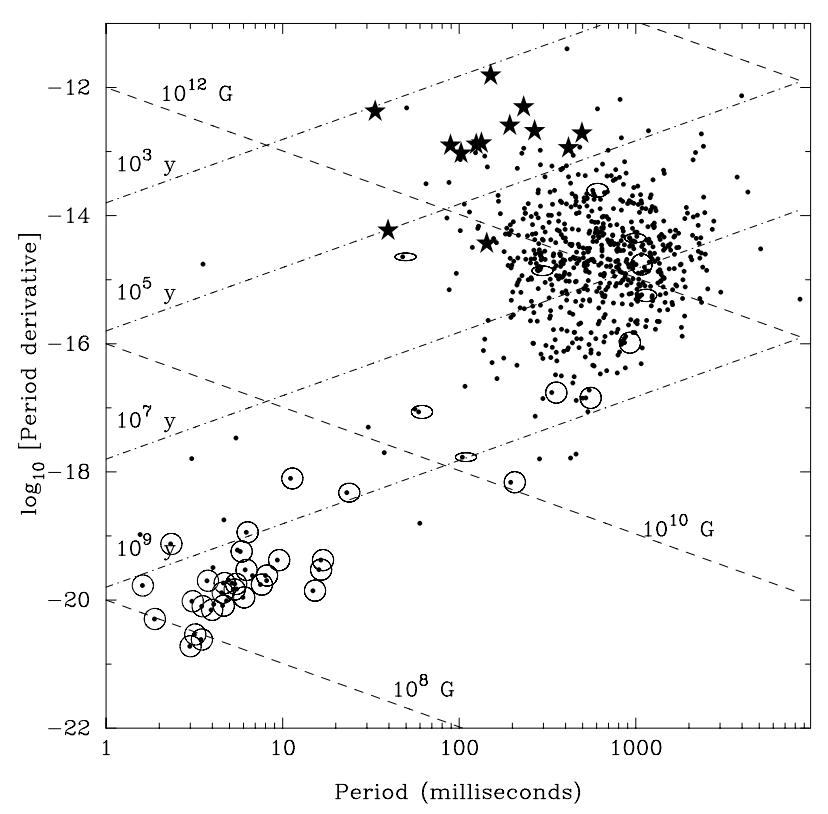

Fig. 3. The location of pulsars on the $P-\dot{P}$ diagram (period-period derivative). Pulsars in binary systems with low-eccentricity orbits are encircled, and in high-eccentricity orbits are marked with ellipses. Stars show pulsars suspected to be connected with supernova remnants [10].

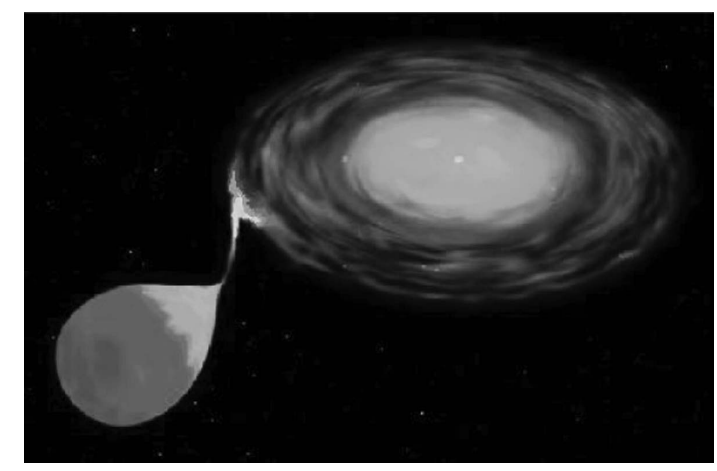

Fig. 4. Artistic vision of a disc accretion to the neutron star in X-ray sources.

\section{A DOUBLE PULSAR SYSTEM}

A binary system containing two pulsars with the periods of 23-ms (J0737-3039 A) and 2.8-sec (B) was discovered in 2004 [14]. This highly-relativistic double-neutronstar system allows unprecedented tests of fundamental gravitational physics. It was observed a short eclipse of J0737-3039 A by J0737-3039 B, and orbital modulation of the flux density and pulse shape of J0737-3039 B, probably due to the influence of J0737-3039A's energy flux upon its magnetosphere. These effects will allow to probe magneto-ionic properties of a pulsar magnetosphere. The observational properties of this system are listed in Table 2, and its artistic view is given in Fig. 5. 


\section{CHECKING GENERAL RELATIVITY}

Five equations in the system (7.2)-(7.6) contain two unknown masses. For correct gravitational theory all curves on the plane $\left(M_{A}, M_{B}\right)$ intersect in the same point. Three curves available for the pulsar $1913+16$ (Fig. 6), and six curves for the double pulsar system (Fig. 7, the additional line $R$ follows from observations of both pulsars) intersect in one point inside the error limits, indicating the correctness of GR on the level $\sim 0.4 \%$ for $1913+16$, after about 20 years of observations, and on the level $\sim 0.1 \%$ after one year of observations of the double system.

\section{VARIABILITY OF THE GRAVITATIONAL CONSTANT}

Variations of the gravitational constant $G$ have been measured by different methods [15]. The following restrictions are obtained from investigations of stellar and planetary orbits. Using the Viking Lander ranging data gave limits $\dot{G} / G=(2 \pm 4) \cdot 10^{-12} \mathrm{yr}^{-1}$. A combination of Mariner 10 and Mercury and Venus ranging gave [16]

$$
\dot{G} / G=(0 \pm 2) \cdot 10^{-12} \mathrm{yr}^{-1}
$$

Lunar Laser Ranging experiments (LLR) have been going on for many years and improved the estimations of $\dot{G} / G$ from $\dot{G} / G<6 \cdot 10^{-12} \mathrm{yr}^{-1}$ in [17], up to $\dot{G} / G$ $\left[\mathrm{yr}^{-1}\right]=(6 \pm 8) \cdot 10^{-13}$ in [18]. Incompletely modeled solid Earth tides, ocean loading or geocenter motion, and uncertainties in values of fixed model parameters have to be considered in those estimations.

Recycled pulsars give the best now available timing precision. The observed changes in the binary period of PSR B1913+16 coincide within error bars with GR prediction of emission of gravitational waves.

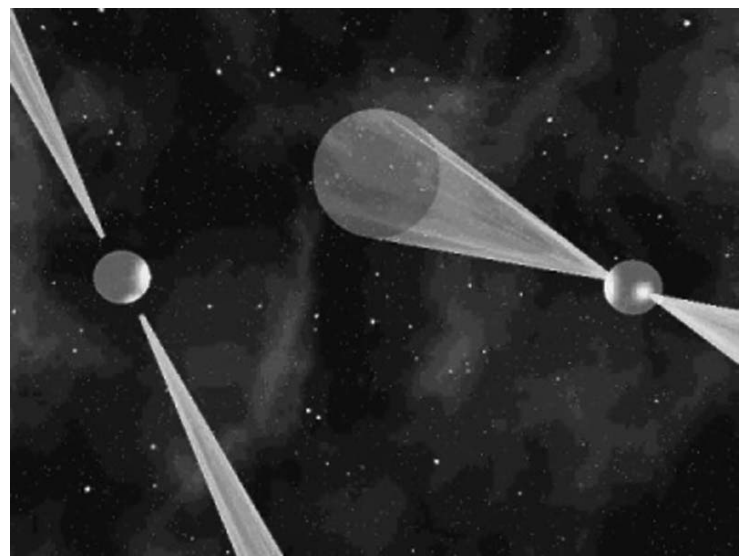

Fig. 5. Artistic picture of the double pulsar system, from [19].

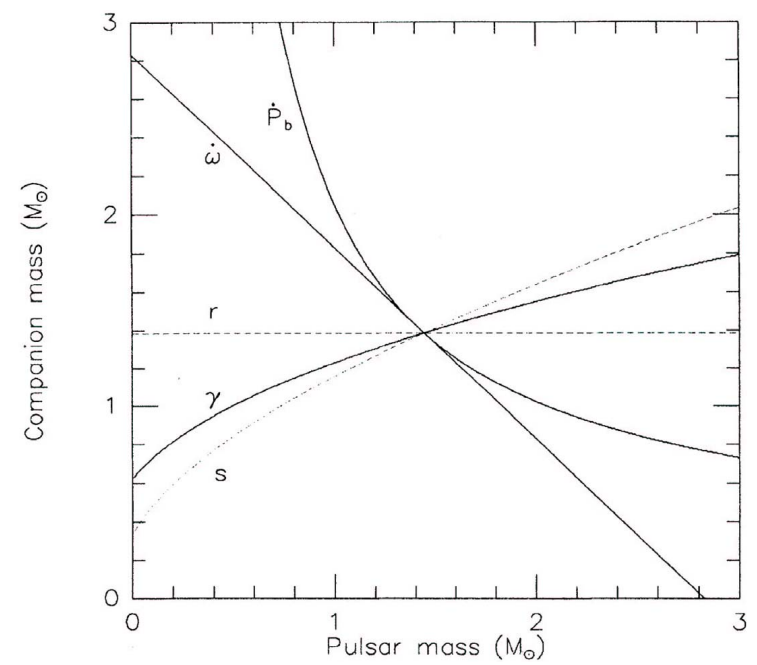

Fig. 6. Observational constraints on the component masses in the binary pulsar PSR $1913+16$. The solid curves correspond to Eqs. (7.2)-(7.4) with measured values of to, $\dot{\omega}, \gamma$ and $\dot{P}_{\text {orb }}$. The intersection of these curves at one point (within an experimental uncertainty of about $0.35 \%$ in $\dot{P}_{\text {orb }}$ ) proves the existence of gravitational waves. The dashed lines correspond to the predicted values of the parameters $r$ and $s$. These values can be measured by future accumulation of observational data [13].

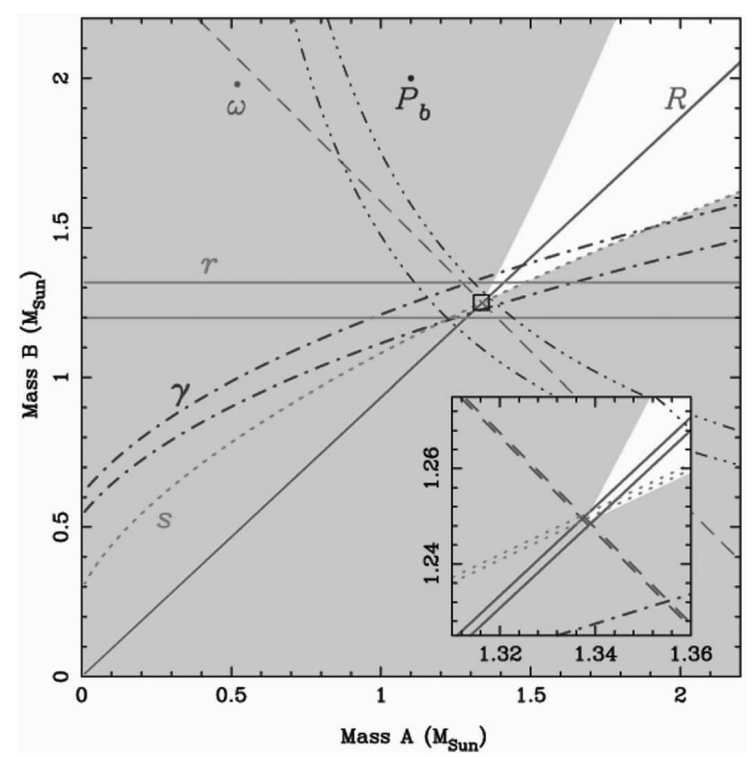

Fig. 7. The observational constraints upon the masses $M_{A}$ and $M_{B}$ in the double pulsar system J0737-3039. The colored regions are those which are excluded by the Keplerian mass functions of the two pulsars. Further constraints are shown as pairs of lines enclosing permitted regions as predicted by general relativity: (a) the measurement of the advance of periastron, giving the total mass $M_{A}+M_{B}=2.588 \pm 0.001 M_{\odot}$ (dashed line); (b) the measurement of $R=M_{A} / M_{B}=1.071 \pm 0.001$ (solid line); (c) the measurement of the gravitational redshift/time dilation parameter (dot-dash line); (d) the measurement of Shapiro parameter $r$ (dot-dot-dot-dash line) and (e) Shapiro parameter $s$ (dotted line). Inset is an enlarged view of the small square encompassing the intersection of these constraints, from [20]. 


\section{G. S. BISNOVATYI-KOGAN}

Measurements of the variations of the binary period give restrictions to the variation of the gravitational constant. Change of $G$ influences on the orbital motion, and solely the emission of gravitational waves compete here with the variation of $G$. If we accept the correctness of GR, then we can use residuals (error box) of the measurements of decay of the binary period $\dot{P}_{b}$ for the estimation of variations of $G$ [21]. It was obtained in [22], using timing data from [23].

$$
\frac{\dot{G}}{G}=-\frac{1}{2} \frac{\dot{P}_{b}}{P_{b}}=(4 \pm 5) \cdot 10^{-12} \mathrm{yr}^{-1} .
$$

For the pulsar PSR B1913+16 the error budget for the orbital period derivative, in comparison with the GR prediction, is given in Table 3 [23]. Supposing that all deviations from GR are connected with $G$ variations, we obtain the upper limit for these variation as follows [24]: $\frac{\dot{G}}{G}=(4.3 \pm 4.9) \cdot 10^{-12} \mathrm{yr}^{-1}$.

\begin{tabular}{|c|c|c|}
\hline Pulsar & PSR J0737-3039A & PSR J0737-3039B \\
\hline Pulse period $P(\mathrm{~ms})$ & $22.699378556138(2)$ & $2773.4607474(4)$ \\
\hline Period derivative $\dot{P}$ & $1.7596(2) \times 10^{-18}$ & $0.88(13) \times 10^{-15}$ \\
\hline Right ascension $\alpha(\mathrm{J} 2000)$ & \multicolumn{2}{|c|}{$07^{\mathrm{h}} 37^{\mathrm{m}} 51^{\mathrm{s}} .24795(2)$} \\
\hline Declination $\delta(\mathrm{J} 2000)$ & \multicolumn{2}{|c|}{$-30^{\circ} 39^{\prime} 40^{\prime \prime} .7247(6)$} \\
\hline Orbital period $P_{b}$ (day) & \multicolumn{2}{|c|}{$0.1022515628(2)$} \\
\hline Eccentricity $e$ & \multicolumn{2}{|c|}{$0.087778(2)$} \\
\hline Advance of periastron $\dot{\omega}\left(\operatorname{deg} \mathrm{yr}^{-1}\right)$ & \multicolumn{2}{|c|}{$16.900(2)$} \\
\hline Projected semi-major axis $x=a \sin i / c(\mathrm{sec})$ & $1.415032(2)$ & $1.513(4)$ \\
\hline Gravitational redshift parameter $\gamma(\mathrm{ms})$ & $0.39(2)$ & \\
\hline Shapiro delay parameter $s=\sin i$ & $0.9995(4)$ & \\
\hline Shapiro delay parameter $r(\mu \mathrm{s})$ & $6.2(6)$ & \\
\hline Orbital decay $\dot{P}_{b}\left(10^{-12}\right)$ & $-1.20(8)$ & \\
\hline Mass ratio $R=M_{A} / M_{B}$ & \multicolumn{2}{|c|}{$1.071(1)$} \\
\hline Characteristic age $\tau$ (My) & 210 & 50 \\
\hline Surface magnetic field strength $B$ (Gauss) & $6.3 \times 10^{9}$ & $1.6 \times 10^{12}$ \\
\hline Spin-down luminosity $\dot{E}(\mathrm{erg} / \mathrm{s})$ & $5800 \times 10^{30}$ & $1.6 \times 10^{30}$ \\
\hline Distance (kpc) & \multicolumn{2}{|c|}{$\sim 0.6$} \\
\hline
\end{tabular}

Table 2. Observed and derived parameters of PSRs J0737-3039A and B. Standard errors are given in parentheses after the values and are in the units of the least significant digit(s). The distance is estimated from the dispersion measure and a model for the interstellar free electron distribution, from $[14,20]$.

These estimations slightly improve the values [22]. The improvement narrows by $40 \%$ the boundary in the negative region, most important from the theoretical point of view. Combining the latter result with [16] permits to narrow the range of $G$ variations, which can now be situated in the limits [24]

$\dot{G} / G$ is within the interval $(-0.6 \div 2) \cdot 10^{-12} \mathrm{yr}^{-1}$.

\begin{tabular}{|l|l|l|}
\hline & Parameter & $\left(10^{-12}\right)$ \\
\hline Observed value & $\dot{P}_{b}^{\text {obs }}$ & $-2.4225 \pm 0.0056$ \\
Galactic contribution & $\dot{P}_{b}^{\text {gal }}$ & $-0.0124 \pm 0.0064$ \\
Intrinsic orbital period decay & $\dot{P}_{b}^{\text {obs }}-\dot{P}_{b}^{\text {gal }}$ & $-2.4101 \pm 0.0085$ \\
General relativistic prediction & $\dot{P}_{b}^{\text {GR }}$ & $-2.4025 \pm 0.0001$ \\
\hline
\end{tabular}

Table 3. Error budget for the orbital period derivative, in comparison with the general relativistic prediction.

\section{MATRE: MOST ACCURATE TIME MEASUREMENTS BY RECYCLED PULSARS (SPACE WATCH)}

Exact time measurements are needed in many branches of human activity, as well as by simple pedestrians.
Nature had prepared for us the most accurate available watch in the form of Recycled Pulsars. The precision of the period of these pulsars is very high, and they are changing their periods with time incredibly slowly: $d P / d t=10^{-19.5}$ for PSR $1953+29, P=6.133 \mathrm{~ms}$; $d P / d t=10^{-19.0}$ for PSR $1937+21, P=1.558 \mathrm{~ms}$, that 
is, for a long time period, much better than the highest available precision based on the hydrogen frequency standard.

I suggest to build the international time watch system based on radio observations of several most stable recycled pulsars. To build this system the following components should be constructed (see Fig. 8).

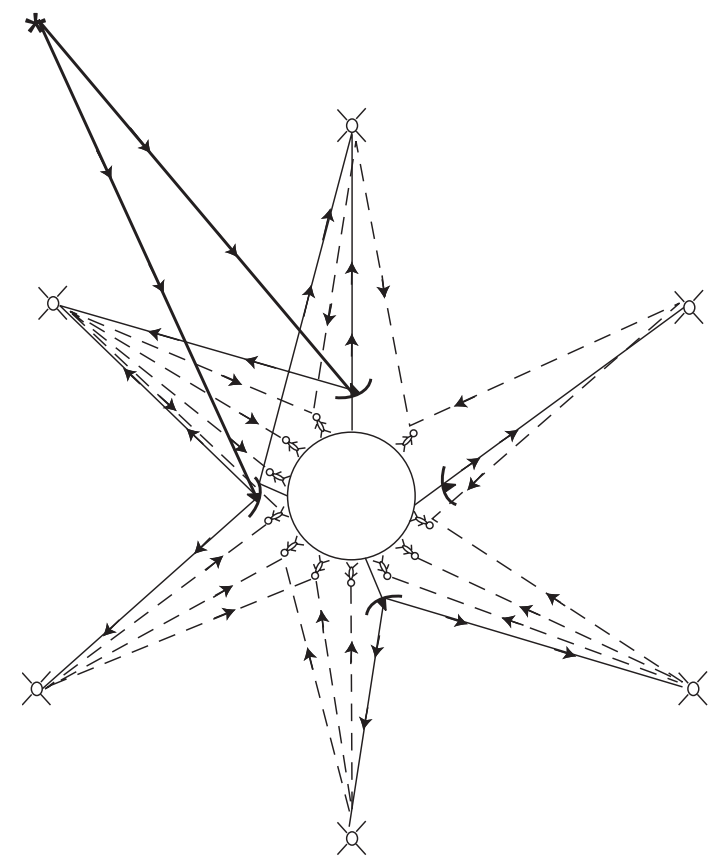

Fig. 8. A schematic sketch of the Space Watch system. The signal from the recycled pulsar (upper left) is accepted by the Earth radio telescopes, which transfer it to the satellite system. The pedestrians accept the exact time signal from the satellite by receivers similar to those used in GPS (or GLONASS).

1. Several radio telescopes over the Earth, which provide stable continuous observations of the chosen pulsar(s) during 24 hours, and retransmission of the signal to the Earth satellites.

2. A system of the Earth satellites (20-25) which accept the exact time signals from the radio telescopes, and retransmit them to the Earth, covering all the Earth surface, similar to GPS.

3. A device which accepts the exact time signal from the satellite and gives the exact local/universal time information (should be widely available, could be combined with the cell phone, or GPS device).

4. Software/hardware for transformation of the pulsar signal into the signal of the exact time measurement on the radio telescopes, satellites, and in every receiving device.

Such a system could be used in all the branches of human activity and available to ordinary citizens. It should be really international, because its work depends on the synchronous and non-interruptive work of all telescopes in different countries. The cost of the construction of this system seems to be quite moderate (comparable with the construction of GPS), but the exact time is needed in many more cases than exact coordinates, simply because the overwhelming majority of people have permanent place of life, or are moving along the well known routes. But watches are used by all of them. Therefore, this system could be quite profitable with time, because having been constructed it needs not much for supporting its work.

\section{CONCLUSIONS}

1. Timing of the pulsars J0737-3039 A/B is the most powerful instrument for the verification of General Relativity due to unprecedented precision of the observations. 2. Recycled pulsars are the most precise available time standards. 3. Checking the physics beyond the standard: $G$-variability is possible by timing of recycled pulsars in close binaries. 4 . The service of the exact time based on continuous observations of recycled pulsars may be constructed and used by pedestrians.

Acknowledgements. This work was partially supported by RFBR grants 05-02-17697A, 06-02-91157 and 06-02-90864, and President's grant for the support of leading scientific schools 10181.2006.2.
[1] A. Hewish et al., Nature 217, 709 (1968).

[2] V. Trimble, M. Rees, in The Crab Nebula. Proc. Int. Astronom. Union. Symp. No. 46, edited by R. D. Davies, F. Graham-Smith (Dordrecht, Reidel, Holland, 1970), p. 273.

[3] D. M. Scott, D. A. Leahy, R. B. Wilson, Astrophys. J. 539, 392 (2000).

[4] G. S. Bisnovatyi-Kogan, B. V. Komberg, Astron. Zh. 51, 373 (1974).

[5] R. A. Hulse, J. H. Taylor, Astrophys. J. Lett. 195, L51 (1975).

[6] J. H. Taylor et al., Asrophys. J. 206, L53 (1976).

[7] G. S. Bisnovatyi-Kogan, B. V. Komberg, Sov. Astron. Lett. 2, 130 (1976).
[8] W. H. T. Vlemmings et al., Mem. Soc. Astron. Ital. 76, 531 (2005).

[9] A. G. Lyne, F. Graham-Smith, Pulsar Astronomy (Cambridge Univ. Press, UK, 1998).

[10] D. R. Lorimer, Living Rev. Relat. 8, 7 (2005); preprint astro-ph/0511258 (2005).

[11] G. S. Bisnovatyi-Kogan, Astrofizika 32, 313 (1990).

[12] G. S. Bisnovatyi-Kogan, Phys. Usp. 49, 53 (2006).

[13] J. H. Taylor, Jr., Rev. Mod. Phys. 66, 711 (1994).

[14] A. G. Lyne et al., Science 303, 1153 (2004).

[15] J.-Ph. Uzan, Rev. Mod. Phys. 75, 403 (2003).

[16] J. D. Anderson et al., Space Sci. Rev. 60, 591 (1992).

[17] J. O. Dickey Science 265, 482 (1994).

[18] J. Mueller, J. G. Williams, S. G. Turyshev, preprint gr- 
qc/0509114 (2005).

[19] J. Arons, talk on KITP Program "Physics of Astrophysical Outflows and Accretion Disks", April 4 - July 29, 2005.

[20] M. Kramer et al., preprint astro-ph/0503386 (2005).

[21] T. Damour, G. W. Gibbons, J. H. Taylor, Phys. Rev.
Lett. 61, 1151 (1988).

[22] V. M. Kaspi, J. H. Taylor, M. F. Ryba, Astrophys. J. 428, 713 (1994).

[23] J. H. Taylor, Class. Quant. Grav. 10, 167 (1993).

[24] G. S. Bisnovatyi-Kogan, Int. J. Mod. Phys. D 15, 1047 (2006); preprint gr-qc/0511072.

\section{ПОДВІЙНІ ПІДКРУЧЕНІ ПУЛЬСАРИ ЯК НАЙТОЧНІША ФІЗИЧНА ЛАБОРАТОРІЯ}

Г. С. Бісноватий-Коган

Інститут космічних досліджень РАН, 84/32, вул. Профсоюзна, Москва, 117997, Росія

Лабораторія теоретичної фізики ім. М. М. Боголюбова

Об'єднаного інституту ядерних досліджень, Дубна, Росія

Обговорено такі питання: 1. Пульсари в тісних подвійних системах. 2. Пульсар Галса-Тейлора. 3. Зруйновані пари пульсарів. 4. Статистика підкручених пульсарів. 5. Вимушене випаровування. 6. Ефекти загальної теорії відносности: нейтронна зірка + нейтронна зірка. 7. Система з двох пульсарів. 8. Перевірка загальної теорії відносности. 9. Непостійність гравітаційної сталої. 10. Космічний годинник. 